\title{
Direct Electrochemical Detection of Estriol in Serum by Polymer Brushes Reinforced with Carbon Nanotubes
}

\author{
Lucas Recco, Bruno P. Crulhas, Caroline R. Basso, Gustavo R. Castro, and Valber A. Pedrosa ${ }^{\mathrm{z}}$ \\ Institute of Bioscience, Department of Chemistry and Biochemistry, UNESP-Botucatu, Botucatu, SP 18607621, Brazil
}

The aim of this study was the development a simple and sensitive sensor for estriol detection in serum based on the combination of multiwalled carbon nanotubes with polymer brushes that provides a nanocomposite with high electrochemical response. Scanning electron microscopy was applied for characterization of the surface morphology of the modified electrodes and cyclic voltammetry, differential pulse voltammetry and electrochemical impedance spectroscopy were used to investigate the electrochemical properties of the proposed electrode. Linearity between oxidation peak current and estriol concentration was obtained ranging from $2.0 \times 10^{-6} \mathrm{M}$ to $15 \times 10^{-6} \mathrm{M}$ with a detection limit of $90 \mathrm{nM}$ and sensitivity was calculated to be $0.15 \mu \mathrm{A} / \mu \mathrm{M}$. The polymer reinforced carbon nanotube displayed high selectivity, good stability and reproducibility, making it suitable for the routine analysis of estriol in clinical use.

() 2016 The Electrochemical Society. [DOI: 10.1149/2.0081608jss] All rights reserved.

Manuscript submitted March 1, 2016; revised manuscript received April 6, 2016. Published April 13, 2016. This paper is part of the JSS Focus Issue on Nanocarbons in Sensing Applications.

Estrogen plays essential role in the development and differentiation of organs, signal transduction control and metabolism adjustment. ${ }^{1}$ Estriol $\left(E_{3}\right)$ is the most abundant estrogen circulating in serum of pregnant women. These effects in women include actions related to the development, neuroendocrine problems in the control of ovulation, preparation for fertilization, and implantation in the reproduction. ${ }^{2}$ Assessment of $\mathrm{E}_{3}$ concentration in serum, plasma and urine is of great value for endocrinological investigation. Thus, the measurement of $\mathrm{E}_{3}$ and its metabolized substances in serum might be useful for the investigation and biochemical monitoring.

For estriol detection, traditional chromatography procedures are most used technique, but they are labor-intensive and time consuming. $^{3-5}$ Other traditional method for monitoring estriol is radioimmunoassay. ${ }^{6,7}$ While this method is reliable and sensitive, it suffers from problems associated with restriction to use it in situ. It is not possible to use this methodology without specially equipped laboratories. Also, these methods have the disadvantages of pretreatment and derivatization. With this in mind we have decide to develop a new approach to detect estriol without any pretreatment by using electrochemical devices.

Electrochemical sensors are one of the most powerful analytical tools that have been developed. The fabrication of electrodes based on conductive polymer reinforced with carbon nanotubes (CNT) has gained great interest in sensing area, and it has been demonstrated that the obtained nanocomposites possess properties of each constituents with a synergistic effect. ${ }^{8-10}$ They have attracted much attention for their electrochemical potential to offer enhanced electrical and mechanical properties. These nanocomposites properties promote electron transfer between electroactive species and electrode surface with good chemical stability, high surface area, and excellent electronic properties. Lately, polymer brushes have emerged as a potential candidate to produce efficient electrodes. It has been used in multiple task which include surface patterning with proteins, ${ }^{11}$ controlled encapsulation of drugs, ${ }^{12}$ control biocatalitic activity, ${ }^{13,14}$ and design of sensor including tumor biomarker, ${ }^{15,16}$ protein, ${ }^{17} \mathrm{pH}$ variation, ${ }^{18}$ glucose detection, ${ }^{19}$ and hormone monitoring. ${ }^{20}$ Which make them universally usable for clinical application.

Herein is described the synergy between multiwalled carbon nanotubes (MWNTs) and poly(vinylimidazole) (PVI) a polymer brush, to construct a modified electrode for estriol detection. A high sensitivity sensor with a good repeatability and reproducibility has been achieved. The sensor performance for real sample analysis was assessed using serum samples spiked with estriol. The use of this nanocomposite

żE-mail: vpedrosa@ibb.unesp.br for diagnostic applications shows great promise to meet the rigorous demands of the clinical laboratory for sensitivity, fast response, and cost-effectiveness.

\section{Experimental}

Reagents and apparatus.-Poly (Vinyl Imidazole) was purchased from Polymer Source. Multi-walls carbon nanotubes (MWNTs), estriol, ascorbic acid, sulfuric acid (98\%), nitric acid (60\%), ethylene glycol, hexadecytrimethyl-ammonium bromide (Mw 364.45), toluene, ethanol, ammonium hydroxide, butanone, hydrogen peroxide, 3-glycidyloxypropyl trimethoxysilane (MW 236.34), oxide titanium indium (ITO) and ferrocene monocarboxylic acid (FcMA) were bought from Sigma-Aldrich. Phosphate buffer PBS $0.1 \mathrm{mM}$ was used as an electrolyte for all electrochemistry experiments. Water used for preparation of all aqueous solutions came from a Millipore Direct-Q water purification system (resistivity, $18 \mathrm{M} \Omega \mathrm{cm}^{-2}$ ).

All electrochemical measurements were performed at room temperature with a potentiostat $\mu$ AutoLabIII (Metrohm, NL). Electrochemical cell consisted of a three-electrode system, which contains a working electrode (PVI-MWNTs), a reference electrode (Ag/AgCl $(3 \mathrm{M} \mathrm{KCl})$ ), and a platinum wire as counter electrode. All measurements were carried out individually at least three times, and error bars are displayed in the following figures. Electrochemical impedance spectroscopy (EIS) was performed at an amplitude of $0.005 \mathrm{~V}$ in the presence of $0.05 \mathrm{M}$ FcMA solution containing $0.1 \mathrm{mM}$ PBS using a $\mu$ Autolab Type III/FRA2 response analyzer (Eco Chemie $\mathrm{BV}$ ) with a frequency range of $0.001-10 \mathrm{~Hz}$ and signal amplitude of $5 \mathrm{mV}$. Allimpedance spectra were fitted to equivalent electrical circuits (Randles circuit) using FRA2 software.

Characterization of surface was done by Scanning electron microscopy (SEM) images taken with a Quanta200 (FEI company) fieldemission scanning electron microscope operating at an acceleration voltage of $20 \mathrm{kV}$.

Electrodes preparation.-The ITO electrodes were chemically modified with PVI-MWNTs brushes using grafting-to method according to following procedure. ${ }^{18,21}$ Briefly, $5 \%(\mathrm{~m} / \mathrm{m})$ of MWNTs were diluted in $100 \mathrm{~mL}$ of ethanol and a solution of $\mathrm{H}_{2} \mathrm{SO}_{4}: \mathrm{HNO}_{3}$ (3:1) were used to hydroxylate the MWNTs, simultaneously PVI was diluted in ethanol to cleave the polymer and mixed with MWNTs solution forming a PVI-MWNTs complex and finally it was mixed and added onto ITO for reaction with epoxy group and spread using a spin coater. The process of adsorption of hybrid film onto ITO was realized in oven in vacuum at $140^{\circ} \mathrm{C}$ for $4 \mathrm{~h}$ and after washed with ethanol. 


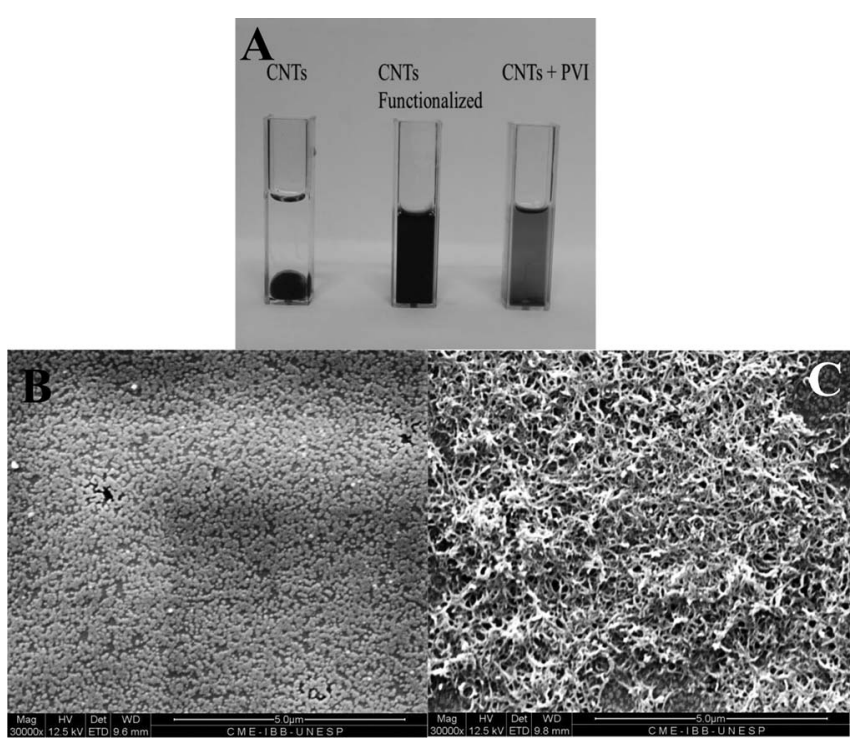

Figure 1. (A) MWNTs-PVI functionalization with Raw MWNTs; MWNTs functionalized in aqueous solution and PVI-MWNTs. (B) Scanning electron microscopy (SEM) of PVI/ITO. (C) Scanning electron microscopy (SEM) of PVI/MWNTs/ITO.

Analysis of spiked serum samples.-The serum samples were analyzed to demonstrate practical application of this methodology. Serum sample was provided by local hospital biochemistry laboratory. Known amounts of the standard estriol solution were added to a $1 \mathrm{~mL}$ aliquot of serum samples, giving a final concentration of 10.0 $\mu \mathrm{mol} \mathrm{L} \mathrm{L}^{-1}$ estriol. No further sample treatment was done. The estriol content was determined by three successive additions of aliquots of the standard estriol solution. Three duplicate experiments were performed for all samples.

\section{Results and Discussion}

Characterization of PVI-CTNs.-The first step to overcome in this work is getting a good dispersion of CNT in polymer solution. Thus, a suspension containing $5 \mathrm{mg}$ of MWNTs were with mixed with $1 \mathrm{~mL}$ of polyvinylimidazole (PVI) to form a well dispersed solution and remained stable for $>2$ months, and showed no evidence of precipitation. Fig. 1A shows MWNTs with high solubility after functionalization, while without this treatment MWNTs were hardly dispersed in water. The success of the strategy was primarily verified by scanning electron microscopy (SEM) characterization. SEM images of the PVI and PVI-MWNTs samples are shown in Figs. 1B and 1C, respectively. Fig. 1B shows a well dispersed polymer layer covering whole ITO surface and Fig. 1C represents the complex MWNTs-PVI demonstrating an enhanced layer with higher surface area when MWNTs was present on top of ITO sensor. Moreover, the PVI film linking the walls of the MWNTs is very smooth and homogeneous, which may enhance the interaction between the modified electrode and further improve the sensitivity and stability of the PVI/MWNTs.

The electrochemical behavior of PVI and PVI-MWNTs electrodes (in $0.1 \mathrm{mM}$ PBS ( $\mathrm{pH} 5.0)$ ) in presence of estriol solution by cyclic voltammetry experiments are shown in Fig. 2A. Redox peaks of estriol were observed at $0.44 \mathrm{~V}$ and $0.26 \mathrm{~V}$ and at $0.42 \mathrm{~V}$ and $0.25 \mathrm{~V}$, for PVI and PVI-MWNTs, respectively. It is observed that the peak current response improved at the PVI-MWNTs with a shift in the peak potential by about $20 \mathrm{mV}$ toward to the less positive values as compared with PVI response. On the basis of these observations, it is clear that addition of MWNTs exerts a significant catalytic effect on
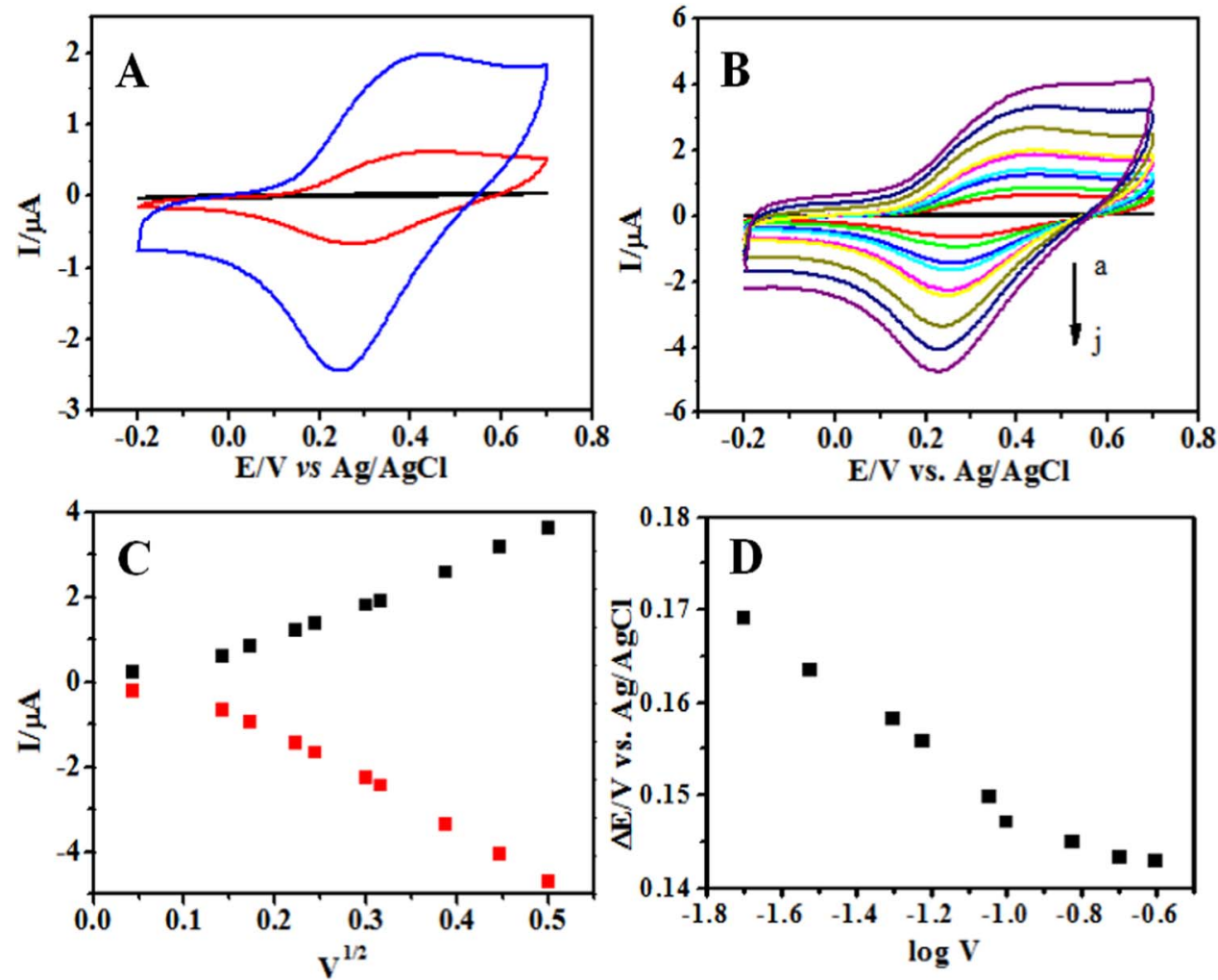

Figure 2. (A) Cyclic voltammograms of bare ITO (black line), PVI/ITO (red line) and PVI/MWNTs/ITO (blue line). (B) Cyclic voltammograms of PVI/MWNTs/ITO electrode at different scan rates (a-j: 20, 30, 40, 50, 60, 70, 100, 150, 200 and $250 \mathrm{mV} / \mathrm{s}$ ) in presence of $0.01 \mathrm{mM}$ of estriol in $0.1 \mathrm{mM}$ PBS buffer ( $\mathrm{pH}$ 5.0). (C) Plot of redox peaks current versus square root of scan rate. (D) Peak-to-peak separation as a function of the potential scan current versus square root of scan rate. 


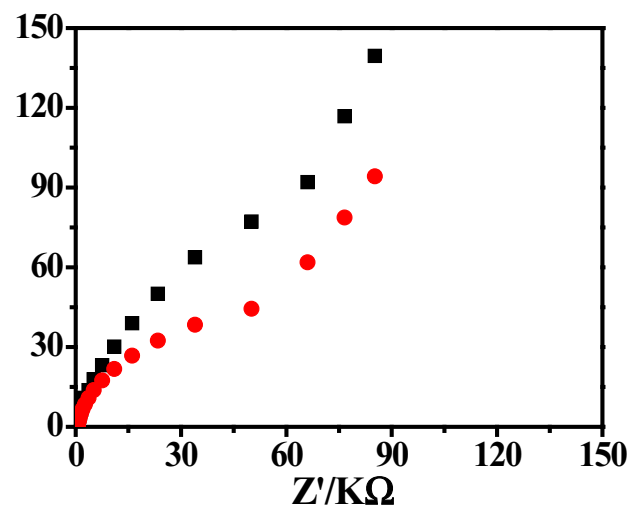

Figure 3. Impedance spectroscopy of PVI/MWNTs/ITO electrode. Nyquist

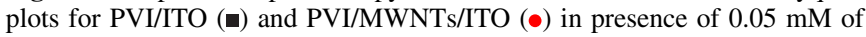
FcMA in $0.1 \mathrm{mM}$ PBS buffer (pH 6.0). Frequency intervals were from $0.1 \mathrm{~Hz}$ to $1 \mathrm{mHz}$ and measurements were carried out at $0.4 \mathrm{~V}$ vs. $\mathrm{Ag} / \mathrm{AgCl}$.

the electrochemical oxidation of estriol leading to decrease of overpotential in the process and enhancement of the peak current is observed. These results suggest that the reason for the better performance of the PVI-MWNTs is due to the electronic structure and topological effects of MWNTs. To characterize voltammetric behavior of PVI-MWNTs, a set of experiments was conducted using cyclic voltammetry. Fig. 2B shows cyclic voltammograms of PVI-MWNTs electrode at different potential scan rates in the presence of $0.05 \mathrm{M}$ FcMA in $0.1 \mathrm{mM}$ PBS buffer. The results obtained shows that with the increase of scan rate, the redox peak current also increase gradually. Correlation of redox peaks currents with scan rate was proportional to square root of the scan rate $\left(v^{1 / 2}\right)$, indicating diffusion controlled transfer reaction (Fig. 2C). The peaks on the cyclic voltammograms were stable and reproducible after many potential cycles and after electrode washing. To better understand this process, the reaction mechanism was analyzed by plotting the peak potential versus scan rate. The peak-topeak $(\triangle E p)$ separation remained constant up to $80 \mathrm{mV}$, suggesting a reversible electrochemical process was occurring on a confined surface (Fig. 2D).

In addition to cyclic voltammetric results, this new nanocomposite was analyzed by electrochemical impedance spectroscopy (EIS), with measurements being performed in the presence of a redox agent (FcMA). EIS can provide information on the impedance changes of electrode surface during modification process. The interface can be modeled by an equivalent circuit, also called a Randles circuit. This equivalent circuit includes the ohmic resistance of electrolyte, $R_{\mathrm{s}}$, the Warburg impedance, $Z_{\mathrm{w}}$, the electron-transfer resistance, $R_{\mathrm{et}}$, and the constant phase element, CPE. In fact, $R_{\mathrm{s}}$ and $Z_{\mathrm{w}}$ are not affected by chemical transformations occurring at electrode interface, since they represent bulk properties of electrolyte solution and diffusion of the applied redox probe. The $C_{\mathrm{dl}}$ changes are not sensitive to the electrontransfer resistance. Fig. 3 compares electrochemical impedance spectra (Nyquist plots, $Z^{\prime \prime}$ vs. $Z^{\prime}$ ) in presence and absence of MWNTs in the polymer network. The diameter of the semicircle represents the charge-transfer resistance $\left(R_{\mathrm{ct}}\right)$ at the electrode surface. As shown in Fig. 3, the diameter of the semicircle, which corresponds to $R_{\mathrm{ct}}$, decreased in presence of MWNTs, suggesting that MWNTs facilitate electron transfer through the film. The calculated values of charge transfer resistance were $\sim 70 \mathrm{~K} \Omega \mathrm{PVI} / \mathrm{ITO}$ prior to modification with MWNTs (black line) and $\sim 50 \mathrm{~K} \Omega \mathrm{PVI} / \mathrm{ITO}$ after modification with MWNTs (red line). This means that the inclusion of MWNTs improved conductivity of PVI network.

Electrochemical detection of estriol.-Differential pulse voltammetry (DPV) was used to measure the estriol oxidation at different concentrations. Fig. 4A show a single peak at $0.50 \mathrm{~V}$ vs. $\mathrm{Ag} / \mathrm{AgCl}$ in $0.1 \mathrm{mM}$ PBS buffer ( $\mathrm{pH}$ 6.0). The dependence of the peak current on
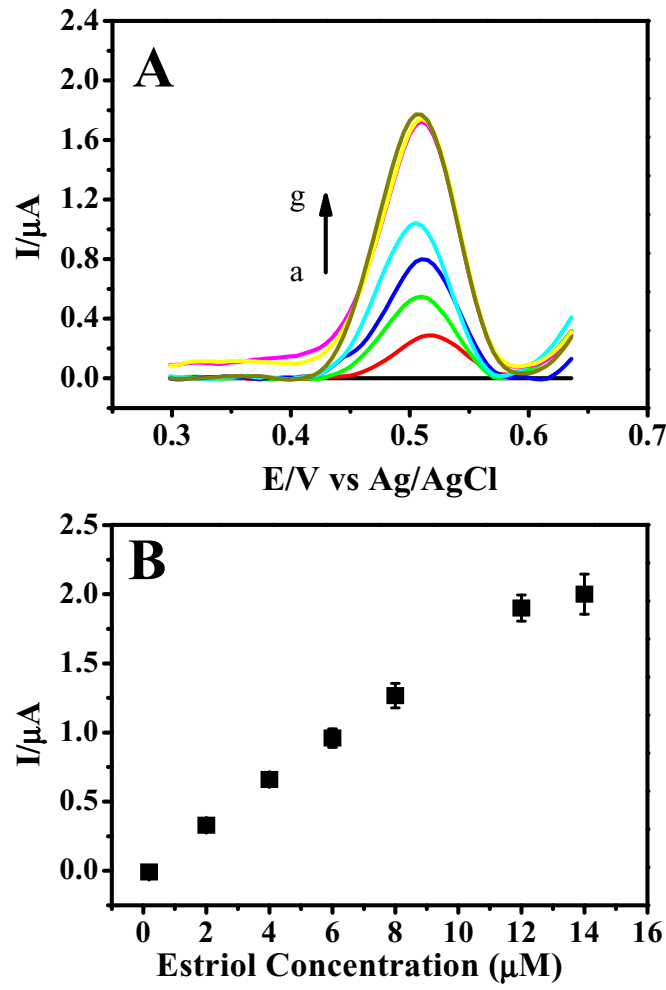

Figure 4. (A) DPV of PVI/MWNTs/ITO at the scan rate of $10 \mathrm{mV} / \mathrm{s}$ in 0.1 $\mathrm{mM}$ buffer and several estriol concentrations (a-g: 0, 2, 4, 6, 8, 12 and 14 $\mu \mathrm{M})$. (B) Calibration plot obtained for PVI/MWNTs/ITO electrode showing absolute value of the current as a function of estriol concentrations.

concentration results in the linear plot shown in Fig. 4B. The linear relationship between Ip and estriol concentration can be described by $\mathrm{I}_{\mathrm{pa}}=0.034 \mu \mathrm{A}+0.15 \mu \mathrm{A} / \mathrm{mmol} \mathrm{L}^{-1}$, and the correlation coefficient was 0.998 . The detection limit $(\mathrm{S} / \mathrm{N}=3)$ for estriol was $90 \mathrm{nM}$, calculate by using a $3 \mathrm{~s} / \mathrm{slope}$ ratio, where $\mathrm{s}$ is the standard deviation of the mean value for 10 voltammograms of the blank, as determined according to IUPAC recommendations. The repeatability is expressed as the RSD (5.1\%) value of the current obtained for five injections of the same concentration of estriol at different times over 7 days by daily testing our sensor. The present methodology shows a detection limit and stability comparable with previous reports..$^{22,23}$

Interference analysis.-The ability to determine estriol in the presence of other organic compound were investigated. The determination of estriol in presence of ascorbic acid (AA) was performed when the concentration of one species changed, whereas the other species remained constant. Fig. 5A shows DPV curves of different concentrations of estriol, in $0.1 \mathrm{mM}$ PBS (pH 6.0), containing $10 \mu \mathrm{M}$ of ascorbic Acid. AA exhibits a well-defined oxidation peak at $0.1 \mathrm{~V}$ vs. $\mathrm{Ag} / \mathrm{AgCl}$, under the same experimental conditions used for estriol determination. The results showed that $i_{\mathrm{pc}}$ was increased proportionality to the concentration of estriol in the range of $2.0 \times 10^{-6}$ to $1.0 \times 10^{-5} \mathrm{~mol} \mathrm{~L}^{-1}$. It can be seen that even in the presence of AA the PVI/MWNTs electrode is sensitive enough to clearly separate the electrochemical responses, indicating that it is possible determinate estriol in the presence of AA.

Analytical applications.-In order to evaluate the validity of the proposed method, estriol was determined in serum samples, without any previous extraction, clean-up or pre-concentration steps. Under same experimental conditions as described above, linear calibration curves were obtained for estriol determination in serum samples, corresponding to linear relationship $\mathrm{I}_{\mathrm{pa}}=0.0034 \mu \mathrm{A}+$ $0.13 \mu \mathrm{A} / \mathrm{mmol} / \mathrm{L}^{-1}$, with a limit of detection of $70 \mathrm{nM}\left(\mathrm{R}^{2}=0.985\right)$ 
Table I. Comparsion of the electrochemical sensors for estriol detection.

\begin{tabular}{llll} 
Material & Technique & LOD $(\mathrm{Mol} / \mathrm{L})$ & Linear Range \\
\hline BBD & SWV & $1.7 \times 10^{-7}$ & $2.0 \times 10^{-7}-2.0 \times 10^{-5}$ \\
MMIPs NPs/CPE & CV & $1.0 \times 10^{-7}$ & $6.0 \times 10^{-7}-1.0 \times 10^{-4}$ \\
LAC/rGO/SB ${ }_{2} \mathrm{O}_{5} / \mathrm{GCE}$ & Amperometry & $1.1 \times 10^{-8}$ & $2.5 \times 10^{-8}-1.03 \times 10^{-6}$ \\
NI-GCE & CV & $1.0 \times 10^{-7}$ & $5.0 \times 10^{-6}-1.0 \times 10^{-4}$ \\
PT/MWNTs/GCE & SWV & $6.2 \times 10^{-7}$ & $1.0 \times 10^{-6}-7.5 \times 10^{-5}$ \\
PPOMC & SWV & $5.0 \times 10^{-9}$ & $1.0 \times 10^{-8}-2.0 \times 10^{-6}$ \\
PVI/CNTs/ITO & DPV & $9.0 \times 10^{-8}$ & $2 \times 10^{-6}-14 \times 10^{-6}$
\end{tabular}

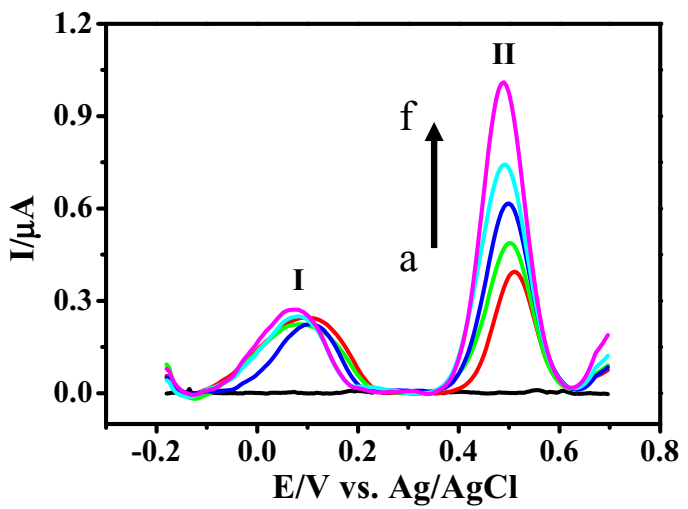

Figure 5. Intereference analysis of PVI/MWNTs/ITO electrode at the scan rate of $10 \mathrm{mV} / \mathrm{s}$ in the presence of $10 \mu \mathrm{M}$ of ascorbic acid as function of estriol concentration (a-f: $0,2,4,6,8$ and $10 \mu \mathrm{M}$ ).

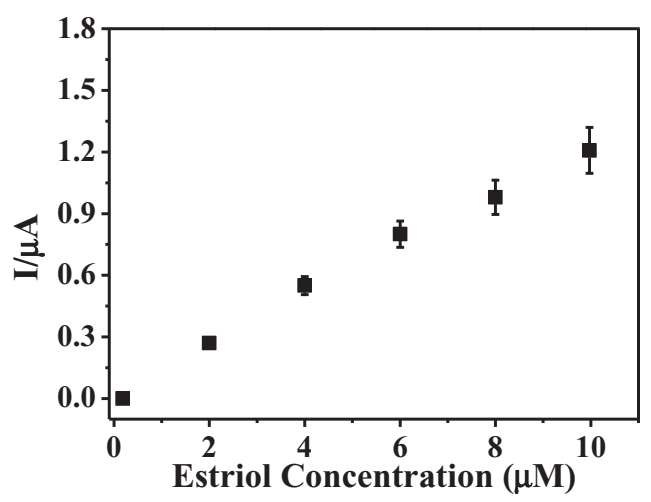

Figure 6. Calibration plot obtained for PVI/MWNTs/ITO electrode, showing absolute value of the current as function of several estriol $(0,2,4,6,8$ and $10 \mu \mathrm{M})$.

(Fig. 6). The recovery experiments were carried out by adding a know amount of estriol $\left(1.0 \times 10^{-7} \mathrm{M}\right)$, minimizing dilution effects $(\leq 1 \%)$ followed by standard additions from the estriol stock solution and plotting the resulting analytical curve solution. All experiments were performed in triplicate. The recovery of all measured samples was between $94.5 \%$ and $96 \%$. These results indicated that the proposed biosensor system could be applied to estriol analysis in real serum samples. Detection of estriol in complex media resulted in lower peak when compared with buffer samples and it can be explain due to unspecific proteins disrupting and interfering with electrochemical signal inside the cell. Nevertheless, even with a lower linear range, the limit of detection remained the same and it can be compared with concentration found in human body. Furthermore, comparison of our device with similar sensors found in literature (Table I), demonstrate a new and robust tool for estriol detection in buffer and complex samples with better stability and sensitivity.

\section{Conclusions}

Our study describes the use of polymer brushes reinforced with carbon nanotubes enabling the detection of estriol. The resulting sensor exhibited good electrochemical response to estriol in PBS and serum sample. It also presented a number of other attractive features such as fast response, high reproducibility, and satisfactory anti-interference ability when compared with traditional chromatographic techniques. Moreover, it was efficient to monitor estriol in serum samples without any pretreatment, and obtained the lowest LOD reported in the literature. The sensor is easier to handle, very stable, reusable, and cost-effective when compared with other sensors.

\section{Acknowledgments}

We gratefully acknowledge partial support by grants from the FAPESP (2014/05653-5), CNPq and CAPES.

\section{References}

1. P. Huang, V. Chandra, and F. Rastinejad, Annu. Rev. Physiol., 72, 247 (2010).

2. H. F. P. Joosten, F. A. A. Van Acker, D. J. Van Den Dobbelsteen, G. J. M. J. Horbach, and E. I. Krajnc, Toxicol. Lett., 151, 113 (2004).

3. M. J. Bartels, K. A. Brzak, and G. A. Bormett, J. Chromatogr. B, 703, 97 (1997).

4. T. Benijts, W. Lambert, and A. De Leenheer, Anal. Chem., 76, 704 (2004).

5. K. Inoue, K. Kato, Y. Yoshimura, T. Makino, and H. Nakazawa, J. Chromatogr. B, 749, 17 (2003).

6. A. Martinetti, E. Seregni, E. Bajetta, G. F. Bolelli, L. Ferrari, S. Massaron, C. Botti, and E. Bombardieri, Int. J. Biol. Mark., 12, 102 (1997).

7. L. Hendrinks, B. Gielen, G. Pottie, V. Haesen, S. Bagyary, and J. Raus, Anal. Chim. Acta, 275, 113 (1993).

8. S. Sánchez, M. Roldán, S. Pérez, and E. Fàbregas, Anal. Chem., 80, 6508 (2008).

9. J. Liu, S. Tian, and W. Knoll, Langmuir, 21(12), 5596 (2005).

10. S. Mantha, V. A. Pedrosa, E. V. Olsen, V. A. Davis, and A. L. Simonian, Langmuir, 26(24), 19114 (2010).

11. S. Tugulu, A. Arnold, I. Sielaff, K. Johnsson, and H. Klok, Biomacromolecules, 6(3), $1602(2005)$

12. A. K. Bajpai, S. K. Shukla, S. Bhanu, and S. Kankane, Prog. Polym. Sci., 33(11), 1088 (2008).

13. T. R. T. A. Antonio, C. R. Basso, M. F. Cabral, and V. A. Pedrosa, Int. J. Electrochem. Sci., 8, 4150 (2013).

14. L. C. Recco, I. Tokarev, S. Minko, and V. A. Pedrosa, Chem. Eur. J., 20, 1226 (2014).

15. X. Wang, M. Zhou, Y. Zhu, J. Miao, C. Mao, and J. Shen, J. Mater. Chem. B, 16, $2132(2013)$.

16. S. Hou, H. Zhao, L. Zhao, Q. Shen, K. S. Wei, D. Y. Suh, A. Nakao, M. A. Garcia, M. Song, T. Lee, B. Xiong, S. C. Luo, H. R. Tseng, and H. H Yu, Adv. Mater. 25, 1547 (2013)

17. Y. Zhao, Y. Zheng, R. Kong, L. Xia, and F. Qu, Biosens. Bioelectron., 75, 383 (2016).

18. T. R. Antonio, M. F. Cabral, I. Cesarino, S. A. Machado, and V. A. Pedrosa Electrochem. Commun., 29, 41 (2013).

19. B. P. Crulhas, J. R. Sempionatto, M. F. Cabral, S. Minko, and V. A. Pedrosa, Electroanal., 26(4), 815 (2014).

20. S. Sánchez, M. Roldán, S. Pérez, and E. Fàbregas, Anal. Chem., 80(17), 6508 (2008)

21. X. Lou, C. Detrembleur, C. Pagnoulle, R. Jérôme, V. Bocharova, A. Kiriy, and M. Stamm, Adv. Mater, 16, 2123 (2004).

22. K. D. Santos, O. C. Braga, I. C. Vieira, and A. Spinelli, Talanta, 80, 1999 (2010).

23. L. L. Zhu, Y. H. Cao, and G. Q. Cao, Biosens. Bioelectron., 54, 258 (2014).

24. A. S. Machado, A. Sánchez, M. A. R. Barrio, R. Villalonga, and J. M. Pingarróna, Electrochim. Acta, 174, 332 (2015).

25. E. Araque, R. Villalonga, M. Gamella, P. Martinez-Ruiz, J. Reviejo, and J. M. Pingarron, J. Mater. Chem. B, 1, 2289 (2013).

26. E. Araque, R. Villalonga, M. Gamella, P. Martinez-Ruiz, A. Sanchez, V. Garcia- Baonza, and J. M. Pingarron, ChemPlusChem., 79, 1334 (2014).

27. A. Boujakhrout, A. Sánchez, P. Díez, S. Jimenez, P. Martínez-Ruiz, M. Peña-Álvarez, J. M. Pingarrón, and R. Villalonga, J. Mater. Chem. B, 3, 3518 (2015). 